\section{Expanding the aptamer alphabet}

\section{By Kai-Jye Lou, Staff Writer}

Researchers at the RIKEN Center for Life Science Technologies and TagCyx Biotechnologies have used an expanded genetic alphabet containing unnatural nucleotides to generate DNA aptamers that have 100fold higher binding affinity for a target protein than counterparts created using only natural nucleotides. ${ }^{1}$ The group plans to develop the aptamers for diagnostic applications before exploring therapeutic applications.

Nucleic acid aptamers consist of single strands of DNA or RNA and are usually generated via in vitro approaches such as SELEX (systematic evolution of ligands by exponential enrichment), in which aptamers from a library undergo iterative rounds of selection to enrich for aptamers with the highest affinity for a particular target.

However, generating molecules with the desired level of functionality usually is less efficient for nucleic acid aptamers than for peptides and proteins. The reason is that a four-nucleotide genetic alphabet limits chemical and structural diversity, according to Ichiro Hirao, leader of the synthetic molecular biology team at the RIKEN Center for Life Science Technologies and founder, president and CEO of TagCyx.

Conventional peptides and proteins are generated using a 20 -aminoacid alphabet.

For more than a decade, Hirao and colleagues in Japan have been developing base pairs of unnatural nucleotides that could be used to expand the standard genetic alphabet and yield oligonucleotides with new properties and functionality. These nucleotide pairs are designed to be compatible with DNA/RNA replication and transcription but only form base pairs with one another and not with any of the four natural nucleotides.

Hirao and colleagues at RIKEN and TagCyx previously reported that the hydrophobic unnatural nucleotide 7-(2-thienyl) Life Science Technologies

imidazo $[4,5-b]$ pyridine (Ds) and its base pair partner, a diol-modified 2-nitro-4-propynylpyrrole, could be incorporated into DNA and were compatible with both SELEX and PCR amplification. ${ }^{2-4}$

Now, Hirao's group has assessed the functional consequences of using an expanded genetic alphabet consisting of four natural nucleotides plus Ds to generate libraries of DNA aptamers. In vitro selection experiments against human VEGF-165 and interferon- $\gamma$ (IFNG; IFN- $\gamma$ ) identified
DNA aptamers from these libraries that bound to the proteins with subpicomolar and subnanomolar $K_{\mathrm{d}}$ values.

Those values represented more than a 100 -fold increase compared with those for DNA aptamers generated using only natural nucleotides.

Results were published in Nature Biotechnology.

"The reported data suggest augmenting standard genetic alphabets with unnatural nucleotides is a feasible approach for expanding the chemical and structural space covered by aptamers and improving their functionality," said Andrew Ellington, a professor of chemistry and biochemistry at The University of Texas at Austin. "They have now opened opportunities to create even larger genetic alphabets that could yield further improvements in aptamer diversity and function."

Noxxon Pharma AG CSO Sven Klussmann thinks Hirao's approach makes sense as a strategy to increase the armamentarium of functionalities available to oligonucleotides. He said adoption of this technology by others in the space will depend on how easy it is to integrate the Ds base with existing experimental methods for aptamer generation and selection.

Ellington noted that previously reported strategies to increase aptamer diversity focused on making modifications to the nucleotides themselves. For example, he said, SOMAmers from aptamer supplier SomaLogic Inc. circumvent the diversity constraints of standard four-nucleotide genetic alphabets by modifying natural nucleotides with hydrophobic functional groups.

Hirao, the paper's corresponding author, thinks expanding the genetic alphabet with unnatural nucleotides is going to be a more efficient approach than modifying natural nucleotides because the former allows access to greater chemical and structural diversity.

He added that the high affinity of the generated aptamers for their targets could mitigate some of the known shortcomings of conventional aptamers such as low in vivo stability and inadequate drug delivery systems.

"Nucleic acid aptamers generally have low stability and are rapidly broken down after getting injected into the body, which could render such a product ineffective," Hirao told SciBX. "By using aptamers with very high binding affinity, the few aptamers that do survive long enough in the body to reach their target might now be sufficient to cause the desired effect. Thus, minimal modification might be sufficient for in vivo stabilization.'

Hirao added that the DNA aptamers generated by his group even had higher binding affinity than known antibodies against the targets-a result that suggests expanding the genetic alphabet has the potential to augment nucleic acids with functionality beyond that of proteins.

"Relative to antibodies, aptamers are also easier to manufacture and modify with functional groups, such as fluorescence dyes," he said.

Noxxon addresses the in vivo stability issue of aptamers by using L-nucleotides to synthesize its aptamer-class molecules, called spiegelmers. L-nucleotides are more resistant to degradation by nucleases than natural D-nucleotides.

Going forward, Klussmann said it would be important to show data on a broad range of molecules that would be easier to target with aptamers using Hirao's method than with other methods. 
Ellington said he wanted to see additional studies exploring the consequences of expanding the genetic alphabet and the crystal structures of aptamers created from such alphabets.

"The unknown is what new structural folds are going to be accessible to nucleic acid aptamers generated with expanded genetic alphabets and how aptamer function is affected," he said. "The expanded alphabet increases structural diversity but might also increase aptamer misfolding."

Noxxon has three lead spiegelmers in development: NOX-E36 inhibits monocyte chemoattractant protein-1 (MCP-1; CCL2) and is in Phase IIa testing to treat diabetic nephropathy; NOX-A12 inhibits chemokine CXC motif ligand 12 (CXCL12; SDF-1) and is in Phase IIa testing for both chronic lymphocytic leukemia (CLL) and multiple myeloma (MM); NOX-H94 inhibits hepcidin and is in Phase IIa testing to treat anemia associated with chronic diseases.

\section{TagCyx tag team}

TagCyx spun out from RIKEN in March 2007 to develop and commercialize products and services that use the unnatural base pair systems being developed at the institute.

In February, the company and partner Dai Nippon Printing Co. Ltd. announced in a press release the development of an anticounterfeiting DNA ink that contains both natural and unnatural nucleotide base pairs.

For the aptamer technology, Hirao said the plan is to develop highaffinity DNA aptamers for research and diagnostic use before exploring therapeutic applications.

"We first would like to develop our aptamers as diagnostic tools that pharmas could use to detect proteins of interest," he said. "We are also exploring the use of these aptamers for purifying populations of induced pluripotent stem cells. We also plan to develop our aptamer technology to go after targets besides proteins, such as small molecules and specific cell types. For therapeutic applications, we also need to develop strategies to stabilize our DNA aptamers against nucleases."

Finally, Hirao noted that the group also is trying to extend the unnatural base pair technology to protein synthesis by using the expanded genetic alphabet to create new codons for unnatural amino acids.

RIKEN and TagCyx have filed multiple patent applications covering the unnatural base pair system and aptamer selection strategy. The technology is available for licensing from TagCyx.

Lou, K.-J. SciBX 6(15); doi:10.1038/scibx.2013.356

Published online April 18, 2013

\section{REFERENCES}

1. Kimoto, M. et al. Nat. Biotechnol.; published online April 7, 2013; doi:10.1038/nbt.2556

Contact: Ichiro Hirao, RIKEN Center for Life Science Technologies, Yokohama, Japan e-mail: ihirao@riken.jp

2. Hirao, l. et al. Nat. Methods 3, 729-735 (2006)

3. Kimoto, M. et al. J. Am. Chem. Soc. 132, 15418-15426 (2010)

4. Yamashige, R. et al. Nucleic Acids Res. 40, 2793-2806 (2012)

5. Davies, D.R. et al. Proc. Natl. Acad. Sci. USA 109, 19971-19976 (2012)

COMPANIES AND INSTITUTIONS MENTIONED

Dai Nippon Printing Co. Ltd. (Tokyo:7912), Tokyo, Japan

Noxxon Pharma AG, Berlin, Germany

RIKEN Center for Life Science Technologies, Yokohama, Japan

SomaLogic Inc., Boulder, Colo.

TagCyx Biotechnologies, Kanagawa, Japan

The University of Texas at Austin, Austin, Texas 